\title{
EPOXI: COMET 103P/HARTLEY 2 OBSERVATIONS FROM A WORLDWIDE CAMPAIGN
}

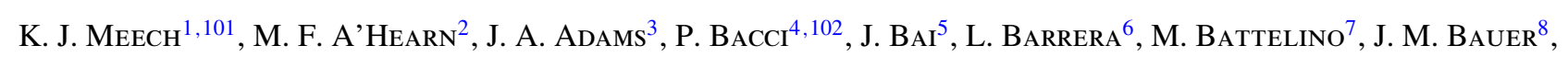
E. BeCKLIN ${ }^{9}$, B. BhatT ${ }^{10}$, N. BIVER ${ }^{11}$, D. Bockelée-Morvan ${ }^{11}$, D. Bodewits ${ }^{2}$, H. BöhnhARdT ${ }^{12}$, J. Boissier ${ }^{13,14}$,

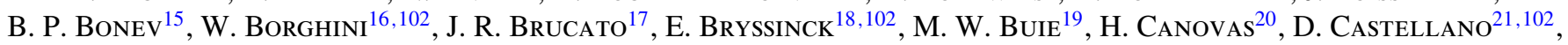
S. B. Charnley ${ }^{22}$, W. P. Chen ${ }^{23}$, P. Chiang ${ }^{24}$, Y.-J. ChOI $^{24}$, D. J. Christian ${ }^{25}$, Y.-L. Chuang ${ }^{26}$, A. L. Cochran ${ }^{27}$, P. Colom $^{11}$,

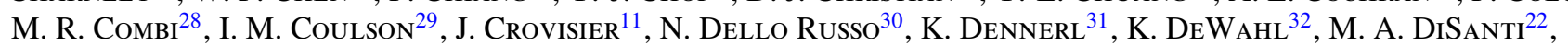
M. FACChini ${ }^{33,102}$, T. L. FARnham ${ }^{2}$, Y. Fernández ${ }^{34}$, H. G. Florén ${ }^{35}$, U. Frisk ${ }^{7}$, T. FuJiYoshi ${ }^{36}$, R. Furusho ${ }^{37}$, T. Fuse ${ }^{38}$,

G. Galli ${ }^{39,102}$, D. A. García-Hernández ${ }^{40}$, A. Gersch ${ }^{2}$, Z. Getu ${ }^{41}$, E. L. GibB ${ }^{42}$, M. Gillon ${ }^{43}$, E. Guido ${ }^{44,102}$,

R. A. Guillermo ${ }^{45}$, E. HadamciK ${ }^{46}$, O. Hainaut ${ }^{14}$, H. B. Hammel ${ }^{47,48}$, D. E. Harker ${ }^{49}$, J. K. Harmon ${ }^{50}$, W. M. Harris ${ }^{51}$,

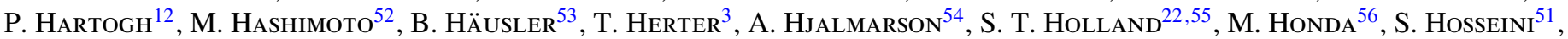
E. S. Howell ${ }^{50}$, N. Howes ${ }^{57,102}$, H. H. Hsieh ${ }^{1}$, H.-Y. HsiaO ${ }^{23}$, D. HutseméKers ${ }^{43}$, S. M. IMMLeR ${ }^{22}$, W. M. JaCKSON ${ }^{51}$, S. V. JefFers ${ }^{20}$, E. Jehin ${ }^{43}$, T. J. Jones ${ }^{32}$, M. De Juan $\operatorname{Ovelar}^{20}$, H. M. Kaluna ${ }^{1}$, T. Karlsson ${ }^{7}$, H. Kawakita ${ }^{58}$, J. V. Keane ${ }^{1}$, L. D. Keller ${ }^{59}$, M. S. Kelley ${ }^{2}$, D. Kinoshita ${ }^{23}$, N. N. $\operatorname{Kiselev}^{60}$, J. Kleyna ${ }^{1}$, M. M. KNight ${ }^{61}$, H. Kobayashi ${ }^{58}$, H. A. KobulnickY ${ }^{62}$, L. Kolokolova ${ }^{2}$, M. KreinY ${ }^{32}$, Y.-J. Kuan ${ }^{26}$, M. KüPPers ${ }^{63}$, J. M. LaCruZ ${ }^{64}$, W. B. LandSMAN ${ }^{22}$,

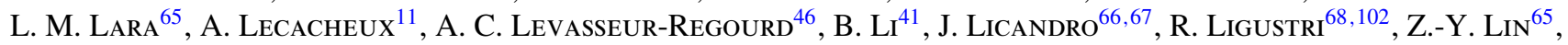

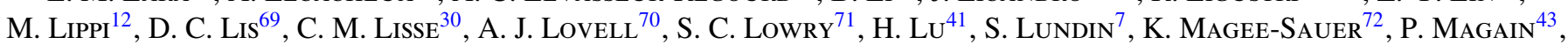
J. Manfroid ${ }^{43}$, E. Mazzotta EpIfani ${ }^{73}$, A. McKaY ${ }^{74}$, M. D. Melita ${ }^{75}$, H. MikUz $^{76,102}$, S. N. Milami ${ }^{22}$, G. Milani ${ }^{77,102}$,

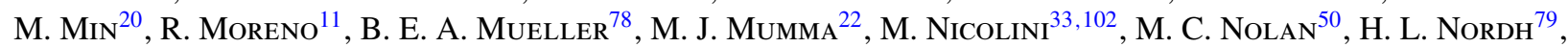
P. B. Nowajewski ${ }^{80}$, Odin Team ${ }^{54}$, T. Ootsubo ${ }^{81}$, L. Paganini ${ }^{22}$, C. Perrella ${ }^{21,102}$, J. Pittichová ${ }^{1}$, E. Prosperi ${ }^{4}, 102$,

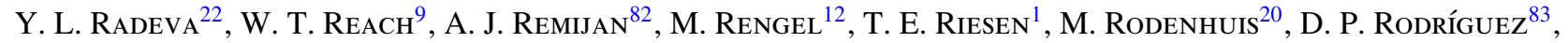

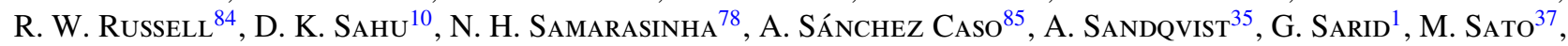
D. G. Schleicher ${ }^{61}$, E. W. Schwieterman ${ }^{61}$, A. K. SeN ${ }^{86}$, D. Shenoy ${ }^{32}$, J.-C. Shi ${ }^{41}$, Y. ShinnakA ${ }^{58}$, J. SkVARC ${ }^{76,102}$, C. Snodgrass ${ }^{12}$, M. L. Sitko ${ }^{49}$, S. SonnetT ${ }^{1}$, S. Sosseini ${ }^{51}$, G. Sostero ${ }^{44,102}$, S. Sugita ${ }^{87}$, B. M. Swinyard ${ }^{88,89}$, S. Szutowicz ${ }^{90}$, N. Takato ${ }^{36}$, P. Tanga ${ }^{91}$, P. A. TaYlor ${ }^{50}$, G.-P. Tozzi ${ }^{17}$, R. Trabatti ${ }^{92,102}$, J. M. Trigo-Rodríguez ${ }^{40}$, C. Tubiana ${ }^{12}$, M. de Val-Borro ${ }^{12}$, W. Vacca ${ }^{9}$, B. Vandenbussche ${ }^{93}$, J. Vaubaillion ${ }^{94}$, F. P. Velichko ${ }^{95}$, S. F. Velichko ${ }^{96}$, R. J. Vervack, JR. ${ }^{30}$, M. J. Vidal-Nunez ${ }^{37}$, G. L. Villanueva ${ }^{22}$, C. Vinante ${ }^{97}$, J.-B. Vincent ${ }^{12}$, M. Wang ${ }^{41}$,

L. H. Wasserman ${ }^{61}$, J. Watanabe ${ }^{37}$, H. A. Weaver ${ }^{30}$, P. R. Weissman ${ }^{8}$, S. Wolk ${ }^{98}$, D. H. Wooden ${ }^{99}$, C. E. Woodward ${ }^{32}$,

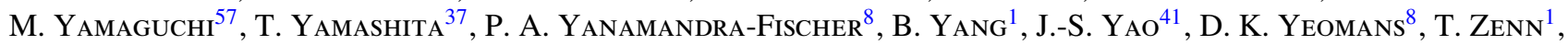
H. ZHAO ${ }^{41}$, AND J. E. ZIFFER ${ }^{100}$

\footnotetext{
${ }^{1}$ Institute for Astronomy, 2680 Woodlawn Drive, Honolulu, HI 96822, USA

2 Department of Astronomy, University of Maryland, College Park, MD 20742-2421, USA

${ }^{3}$ Department of Astronomy, Cornell University, 202 Space Sciences Building, Ithaca, NY 14853, USA ${ }^{4}$ Centro Astronomico di Libbiano, Peccioli Pisa, Italy

${ }^{5}$ Yunnan Astronomical Observatory, Chinese Academy of Sciences, P.O. Box 110, Kunming 65011, Yunnan, China

${ }^{6}$ Universidad Metropolitana de Ciencias de la Educación, Av. J. P. Alessandri 774, Nunoa, Santiago, Chile ${ }^{7}$ Swedish Space Corporation, P.O. Box 4207, 17104 Solna, Sweden 8 Jet Propulsion Laboratory, 4800 Oak Grove Drive, Pasadena, CA 91109, USA

${ }^{9}$ Stratospheric Observatory for Infrared Astronomy, Universities Space Research Association, Mail Stop 211-3, Moffett Field, CA 94035, USA

${ }^{10}$ Indian Institute of Astrophysics, CREST Campus, Block-II, Koramangala, Sarjapur Road, Bangalore 560034, India

${ }^{11}$ LESIA, Observatoire de Paris, 5 place Jules Janssen, Meudon 92195, France

${ }^{12}$ Max-Planck Institute for Solar System Research, Max-Planck-Str. 2, Katlenburg-Lindau 37191, Germany

${ }^{13}$ INAF-Inst. di Radioastronomia, Via P. Gobetti 101, 40129 Bologna, Italy

${ }^{14}$ ESO, Karl-Schwarzschild-Str. 2, 85748 Garching bei München, Germany

${ }^{15}$ Department of Physics, Catholic University of America/GSFC, 620 Michigan Ave., N.E., Washington, DC 20064, USA

${ }^{16}$ Osservatorio Astronomico Naturalistico di Casasco, Strada Ca'Simoni 15050, Casasco, Italy

${ }^{17}$ INAF-Osservatorio Astrofisico di Arcetri, Largo E. Fermi 5, Firenze 50125, Italy

${ }^{18}$ Brixiis Observatory, Eyckensbeekstraat 2, 9150 Kruibeke, Belgium

${ }^{19}$ Department of Space Studies, Southwest Research Institute, 1050 Walnut Street 400, Boulder, CO 80302, USA

${ }^{20}$ Astronomical Institute, Utrecht University, Princetonplein 5, 3584 CC Utrecht, The Netherlands

${ }^{21}$ Unione Astrofili Italiani, IASF INAF via Fosso del Cavaliere 100-00133 Roma, Italy

${ }^{22}$ NASA GSFC, 8800 Greenbelt Rd., Greenbelt, MD 20771, USA

${ }^{23}$ Graduate Institute Astronomy, National Central University, 300 Jhongda Rd, Jhongli 32001, Taiwan

${ }^{24}$ Korea Astronomy and Space Science Institute, Daejeon 305-348, Republic of Korea

${ }^{25}$ Department of Physics and Astronomy, California State University, Northridge, CA 91330, USA

${ }^{26}$ Departmnet of Earth Sciences, National Taiwan Normal University, 88, Sec. 4, Ting-Chou Road, Taipei 11677, Taiwan

${ }^{27}$ McDonald Observatory, University of Texas, 1 University Station, C1402, Austin, TX 78712, USA

${ }^{28}$ Department of Atm. Ocean and Space Sci., 2418B Space Research Bldg., University of Michigan, 2455 Hayward St., Ann Arbor, MI 48109-2143, USA

${ }^{29}$ Joint Astronomy Centre, 660 N. Aohoku Place, University Park, Hilo, HI 96720, USA

30 JHU/APL, 11100 Johns Hopkins Road, Laurel, MD 20723, USA

${ }^{31}$ Max-Planck-Institut für Extraterrestrische Physik, Giessenbach-strasse, 85748 Garching, Germany

${ }^{32}$ Department of Astronomy, University of Minneapolis, 116 Church St. SE, Minneapolis, MN 55455, USA

${ }^{33}$ G. Montanari Observatory, via Concordia 200, 41032 Cavezzo, Italy

${ }^{34}$ Department of Physics, University of Central Florida, 4000 Central Florida Blvd., Orlando, FL 32816-2385, USA

${ }^{35}$ Stockholm Observatory, SCFAB AlbaNova, 10691 Stockholm, Sweden

${ }^{36}$ Subaru Telescope, National Astronomical Observatory of Japan, 650 North A'ohoku Place, Hilo, HI 96720, USA
} 
${ }^{37}$ National Astronomical Observatory of Japan, Osawa 2-21-1, Mitaka, Tokyo 181-8588, Japan

${ }^{38}$ Kashima Space Research Center, NICT, Ibaraki 314-8501, Japan

${ }^{39}$ GiaGa Observatory, Pogliano Milanese, Milano, Italy

${ }^{40}$ Institute of Space Sciences (CSIC-IEEC), Campus UAB, Sciences Faculty, Tower C-5, Even, 2nd floor, Bellaterra (Barcelona) E-08193, Spain

${ }^{41}$ Purple Mountain Observatory, 2 West Beijing Road, Nanjing 210008, China

42 Department of Physics and Astronomy, University of Missouri-St. Louis, 503 Benton Hall, One Univ. Blvd., St Louis, MO 63121, USA

${ }^{43}$ Institut d'Astrophysique et de Géophysique, Université de Liège, Allée du 6 Août, 17, 4000 Liège, Belgium

${ }^{44}$ Remanzacco Observatory, via S. Stefano, 33047, Remanzacco, Italy

${ }^{45}$ Depto. de Física, Facultad de Ciencias, Universidad de Chile, Las Palmeras 3425, Ñuñoa, Casilla 653, Santiago, Chile

${ }^{46}$ UPMC Univ. Paris 06/LATMOS-IPSL, 11 Blvd. d'Alembert, 78280 Guyancourt, France

${ }^{47}$ AURA, 1212 New York Ave NW, Suite 450, Washington, DC 20005, USA

${ }^{48}$ Space Science Inst., 4750 Walnut Street, Suite 205, Boulder, CO 80301, USA

${ }^{49}$ Center for Astrophysics \& Space Sciences, University of California, San Diego, 9500 Gilman Dr. Dept 0424, La Jolla, CA 92093-0424, USA ${ }_{50}$ Arecibo Observatory, HC3 Box 53995, Arecibo, PR 00612, USA

${ }^{51}$ University of California, Department of Applied Sciences, One Shields Ave., Davis, CA 95616, USA

52 Saga Pref. Space and Science Museum, 16351, Nagashima, Takeo 843-0021, Japan

53 Maidbronn Observatory, Albin-Joerg-Str. D-97222, Maidbronn, Germany

${ }^{54}$ Onsala Space Observatory, 43992 Onsala, Sweden

${ }^{55}$ Centre for Research and Exploration in Space Science and Technology, Code 668.8, 8800 Greenbelt Road, Goddard Space Flight Center, Greenbelt, MD 20771, USA

${ }^{56}$ Department of Information Sciences, Kanagawa University, Kanagawa 259-1293, Japan

${ }^{57}$ LCOGT/Faulkes Telescope North \& Cherhill Observatory, UK

${ }^{58}$ Department of Physics, Facility of Science Kyoto Sangyo University, Motoyama, Kamigamo, Kita-ku, Kyoto 603-8555, Japan ${ }^{59}$ Ithaca College, Ithaca, NY 14850, USA

${ }^{60}$ Main Astronomical Observatory of National Academy of Sciences of Ukraine, Zabolotnoho 27, Kiev 03680, Ukraine

${ }^{61}$ Lowell Observatory, 1400 W. Mars Hill Road, Flagstaff, AZ 86001, USA

${ }^{62}$ Department of Physics \& Astronomy, University of Wyoming, Laramie, WY 82071, USA

${ }^{63}$ Rosetta Science Operations Centre, European Space Astronomy Centre, European Space Agency, Madrid, Spain

${ }^{64}$ La Cañada Observatory, c/Ramón Gómez de la Serna 83, 7-F, Madrid 28035, Spain

${ }^{65}$ IAA-CSIC, c/Glorieta de la Astrónoma s/n, Granada 18008, Spain

${ }^{66}$ Instituto de Astrofísica de Canarias, c/Via Lactea s/n, 38200 La Laguna, Tenerife, Spain

${ }^{67}$ Depto. de Astrofísica, Univ. de La Laguna, E-38205 La Laguna, Tenerife, Spain

${ }^{68}$ Talmassons Observatory (C.A.S.T.), via Cadorna, 33030 Talmassons, Italy

${ }^{69}$ Caltech, MC 301-17, Pasadena, CA 91125, USA

${ }^{70}$ Department of Physics and Astronomy, Agnes Scott College, Decatur, GA 30030, USA

${ }^{71}$ Centre for Astrophysics and Planetary Science, University of Kent, Canterbury, CT2 7NH, UK

72 Department of Physics and Astronomy, Rowan University, Glassboro, NJ 08028-1701, USA

${ }_{73}$ INAF-OAC, Via Moiariello 16, Napoli 80131, Italy

${ }^{74}$ Department of Astronomy New Mexico State University, P.O. Box, 20001, MSC 4500 Las Cruces, NM 88003-8001, USA

${ }^{75}$ Instituto de Astronomía y Física del Espacio, Buenos Aires, Argentina

${ }^{76}$ Astronomski obsevatorij, Predgriže 29a, 5274 Črni Vrh nad Idrigo, Črni Vrh, Slovenia

77 Dip. di Scienze Biomediche Sperimentali, Universita' di Padova, viale G. Colombo 3, 35121, Italy

${ }^{78}$ Planetary Space Institute, 1700 E. Ft. Lowell, 106, Tucson, AZ 85719, USA

${ }^{79}$ Swedish National Space Board, P.O. Box 4006, 17104 Solna, Sweden

${ }^{80}$ Astronomy Department, University of Chile, Camino el Observatorio \#1515, Las Condes, Santiago, Chile

${ }^{81}$ Astronomical Institute, Graduate School of Science, Tohoku University, Aramaki, Aoba-ku, Sendai 980-8578, Japan

${ }^{82}$ NRAO, 520 Edgemont Road, Charlottesville, VA 22903, USA

${ }^{83}$ Guadarrama Observatory, c/San Pablo 5, Alpedrete, Madrid 28400, Spain

${ }^{84}$ The Aerospace Corp., M2-266, P.O. Box 92957, Los Angeles, CA 90009-2957, USA

${ }^{85}$ Gualba Astronomical Observatory, Mas Cortinas-52, Gualba, Barcelona 08474, Spain

${ }^{86}$ Department of Physics, Assam University, Silchar 788001, India

${ }^{87}$ Department of Complexity Science \& Engineering, University of Tokyo, Kashiwanoha, Kashiwa, Chiba 277-8561, Japan

${ }^{88}$ Planets and Space Plasmas Group, Space Science and Technology Department, Rutherford Appleton Laboratory, Oxfordshire, UK

${ }^{89}$ Department of Physics and Astronomy, University College London, Gower Place, London \#WC1E6BT, UK

${ }^{90}$ Space Research Centre, Polish Academy of Science, Warszawa, Poland

${ }^{91}$ UMR 6202 Cassiopée, University of Nice-Sophia Antipolis, CNRS, Observatoire de la Côte d'Azur, BP 4229, 06304 Nice Cedex 4, France

${ }^{92}$ Stazione Astronomica Descartes, via Lambrinia 4, 27013, Chignolo Pó, Italy

${ }^{93}$ Institute for Astronomy, KU, Leuven, Belgium

${ }^{94}$ IMCCE-Observatoire de Paris, 77 Avenue Denfert-Rochereau, F-75014 Paris, France

${ }^{95}$ Astronomical Institute of Kharkiv University, Sums'ka 35, Kharkiv 61022, Ukraine

${ }^{96}$ International Center for Astronomical, Medical and Ecological Research, 27 Akademika Zabolotnoho, Kiyv 03680, Ukraine

${ }^{97}$ Dipartimento di Geoscienze, Università di Padova via Gradengio, 6-35131 Padova, Italy

${ }^{98}$ Chandra X-Ray Center, Harvard-Smithsonian Center for Astrophysics, 60 Garden Street, Cambridge, MA 02138, USA

${ }_{99}$ NASA-Ames Research Center, MS 245-3, Moffett Field, CA 94035-0001, USA

${ }^{100}$ University of Southern Maine, 96 Falmouth Street, Portland, ME 04104-9300, USA

Received 2011 March 4; accepted 2011 April 20; published 2011 May 16

\section{ABSTRACT}

Earth- and space-based observations provide synergistic information for space mission encounters by providing data over longer timescales, at different wavelengths and using techniques that are impossible with an in situ flyby. We report here such observations in support of the EPOXI spacecraft flyby of comet 103P/Hartley 2. The nucleus is small and dark, and exhibited a very rapidly changing rotation period. Prior to the onset of activity, the period 
was $\sim 16.4 \mathrm{hr}$. Starting in 2010 August the period changed from $16.6 \mathrm{hr}$ to near $19 \mathrm{hr}$ in December. With respect to dust composition, most volatiles and carbon and nitrogen isotope ratios, the comet is similar to other Jupiter-family comets. What is unusual is the dominance of $\mathrm{CO}_{2}$-driven activity near perihelion, which likely persists out to aphelion. Near perihelion the comet nucleus was surrounded by a large halo of water-ice grains that contributed significantly to the total water production.

Key words: comets: individual (103P/Hartley 2)

\section{INTRODUCTION}

Comet $103 \mathrm{P} /$ Hartley 2, the EPOXI mission target, was discovered in 1986, and at the time of mission selection, had been observed at four apparitions. The comet is on a $6.47 \mathrm{yr}$ period orbit, with a perihelion distance of $q=1.05 \mathrm{AU}$ and aphelion distance of $Q=5.88$ AU. It made a close approach to Jupiter in 1971 to $0.1 \mathrm{AU}$, an event that changed the perihelion distance from 1.48 to 0.9 AU. Prior to 1971, back to 1900 at least, the perihelion distance was about 1.4 AU. An Earth- and space-based campaign was initiated in 2008 to complement the in situ mission. The purpose of the campaign was to collect data over timescales, at wavelengths, and with instruments not carried on the EPOXI mission, to help characterize the comet pre-encounter and to provide scientific context for the interpretation of the in situ data. Observers were coordinated via an email list-server and a central Web site that enabled the group to rapidly share information about the comet brightness and activity necessary for observation planning during the several months around encounter. During the full campaign, which involved the collaboration of nearly 200 registered astronomers (Figure 1), more than 500 whole/partial nights worldwide were awarded on 51 telescopes involving 11 countries, 8 space facilities (Hubble Space Telescope (HST), Spitzer, Widefield Infrared Survey Explorer (WISE), Swift, ODIN, Chandra, Solar and Heliospheric Observatory (SOHO), Herschel) and the Stratospheric Observatory for Infrared Astronomy (SOFIA) airborne observatory.

\section{NUCLEUS PROPERTIES AND ROTATION}

The comet was first seen after its aphelion passage (2007 July) using the European Southern Observatory (ESO) Very Large Telescope (VLT) in Chile while it was at 5.65 AU during 2008 May $(Q+272$ days). It showed activity after passing aphelion that faded over the course of the following three months (Snodgrass et al. 2010). Observations with the Spitzer Space Telescope during 2008 August indicated a small average effective radius of $0.57 \pm 0.08 \mathrm{~km}$ with a reported geometric albedo (at $0^{\circ}$ phase) of $0.028 \pm 0.009$ and an extended dust trail which modeling found to be composed of large (millimetersized) particles produced during the previous perihelion passage (Lisse et al. 2009).

Data were obtained during 2009 April-May using the HST and Gemini $8 \mathrm{~m}$ telescopes. The nucleus rotation periodicity was measured to be $16.4 \pm 0.2 \mathrm{hr}$, with no apparent activity. This same periodicity was also reported in 2010 August from enhanced CN filter images $(0.38 \mu \mathrm{m})$ of the comet (see Table 1). The CN morphology evolved throughout the apparition (Figure 2(a)), and this was used to estimate the rotation rate, yielding a longer periodicity, which was consistent with

\footnotetext{
101 Author to whom any correspondence should be addressed.

102 CARA, Unione Astrofili Italani, vicolo Osservatorio 2, 35122, Padova, Italy
}

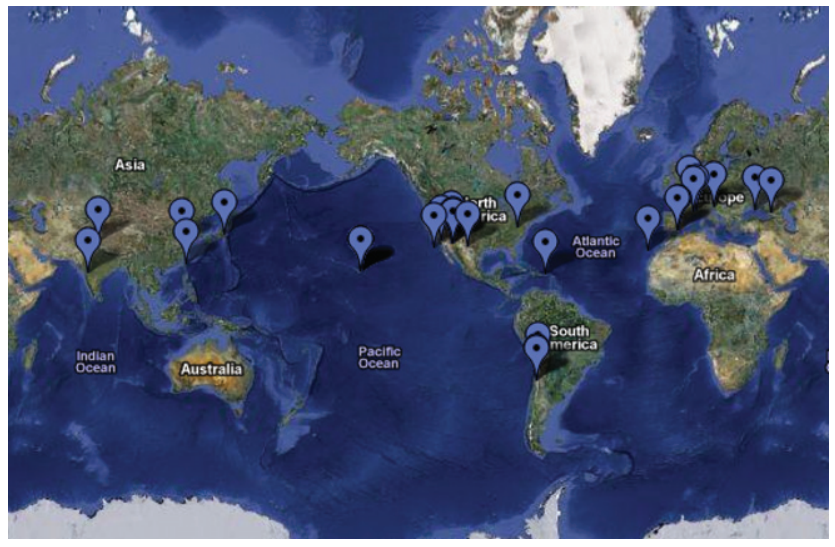

Figure 1. Locations of observatories contributing to the Earth-based campaign.

what was seen by the EPOXI spacecraft (A'Hearn et al. 2011). The new robotic TRAPPIST $60 \mathrm{~cm}$ telescope on La Silla was used to monitor the comet from 2010 October 30 through 2011 January 28 with narrowband cometary and broadband filters (Jehin et al. 2010). The data show periodic flux variations in gas production (also seen by others), with the largest variations observed in the $\mathrm{CN}$ filter. A period of $18.4 \pm 0.3 \mathrm{hr}$ was seen during the first half of 2010 November, in agreement with an $18.1 \pm 0.3 \mathrm{hr}$ synodic periodicity determined from Arecibo Observatory Doppler radar imaging observations obtained during 2010 October 24-27 (Harmon et al. 2010). It then slowed to nearly $19 \mathrm{hr}$ during the second half of 2010 November (Jehin et al. 2010), presumably as a result of the strong jet activity seen by observers (Figure 2(a)) on such a small nucleus (A'Hearn et al. 2011). This relatively quick change in rotation period has rarely been seen in comets, but apparently is also happening with 9P/Tempel 1 but at a factor of $\sim 10$ slower (Belton et al. 2011).

\section{GAS SPECIES}

Narrowband optical imaging, X-ray, UV, optical, near- and mid-IR spectra, submillimeter and radio observations were used to monitor the comet for gas production rates beginning in 2010 July. Late 2010 October through mid-November, Chandra observations of the comet show that X-rays were emitted due to solar wind charge exchange between highly charged $\mathrm{C}, \mathrm{N}$, $\mathrm{O}$, and $\mathrm{Ne}$, highly charged minor ions in the solar wind and neutral gas in the comet's coma. The outgassing rate of the comet was low enough that the overall rate of X-ray emission was the second lowest ever recorded and occurred in regions within $\sim 10^{4} \mathrm{~km}$ of the nucleus. Except during the latter part of November the X-ray emission spectrum seen was typical of the cold, dense, slow equatorial solar wind interacting with a cometary coma.

The Swift satellite observed the comet several times on 2010 September 15 and November 21, and acquired mediumresolution grism spectra and broadband UV-optical imaging. On September 15 during the $\mathrm{CN}$ anomaly observed by EPOXI 
Table 1

Comet 103P/Hartley 2's Changing Rotation Period

\begin{tabular}{lclr}
\hline \hline \multicolumn{1}{c}{ Time Interval } & Period (hr) & \multicolumn{1}{c}{ Measurement Technique } & Ref. \\
\hline 2009 Apr-May & $16.4 \pm 0.1$ & Nucleus light curve, $R$ band & 1 \\
2010 Aug. 13-17 & $16.6 \pm 0.5$ & CN jet imaging & 2 \\
2010 Sept 1-3 & 17.1 & CN jet imaging & 3 \\
2010 Sept 15, 2010 & $17 \& 29^{\text {a }}$ & Spacecraft data & 4 \\
2010 Sept 30-Oct 4 & 17.6 & CN jet imaging; radio observations & 3 \\
2010 Oct 27 & $18.1 \pm 0.3$ & Doppler radar imaging & 5 \\
2010 Oct 29-Dec 2 & $18.4 \pm 0.3$ to 19 & Narrowband photometry (CN) & 6 \\
\hline
\end{tabular}

Notes.

a The spacecraft data started to show evidence of complex rotation with a precession of $17 \mathrm{hr}$ and a second frequency near $29 \mathrm{hr}$.

References. (1) Meech et al. 2009; (2) Knight et al. 2010; (3) Drahus et al. 2011, Samarasinha et al. 2010; (4) A'Hearn et al. 2011; (5) Harmon et al. 2010; (6) Jehin et al. 2010.

(A'Hearn et al. 2011) the $\mathrm{OH}$ production rates were between $1.1 \times 10^{27} \mathrm{~s}^{-1}$ and $2.1 \times 10^{27} \mathrm{~s}^{-1}$, with a value of $A(\theta) f \rho=$ $45 \mathrm{~cm}$ (a proxy for dust production; A'Hearn et al. 1984) and a tentative ratio $\mathrm{C}_{2} / \mathrm{CN}=0.4$. This is much lower than the ratio $\mathrm{C}_{2} / \mathrm{CN}=1.2-1.3$ seen in most comets (A'Hearn et al. 1995). The $\mathrm{CN}$ anomaly is therefore not coupled to either $\mathrm{OH}$ or $\mathrm{C}_{2}$. Simultaneously with the UV observations, Swift used its X-ray telescope but did not detect the comet. Having a much smaller collecting area than Chandra, the non-detection is not surprising given that this was one of the faintest comets ever observed by Chandra. Visible and near-UV photometry obtained four nights prior to encounter yielded a total water production rate of $1.1 \times 10^{28} \mathrm{~s}^{-1}$ and a value of $A(\theta) f \rho=$ $63 \mathrm{~cm}$. These and values obtained earlier in the apparition appear to be consistently lower (by up to $\sim 3 x$ ) than measurements made during the 1991 and 1997 apparitions. However, current photometry confirms the very low dust-to-gas ratio measured during the 1990s and also confirms the abundance ratios of minor species obtained previously; in particular, $\mathrm{C}_{2} / \mathrm{CN}=1.3$ corresponds to a classification of typical cometary composition (A'Hearn et al. 1995). At the time of encounter HST obtained UV spectroscopic observations and detected emission from $\mathrm{CO}$ with a production rate of $Q(\mathrm{CO})=(2.2-2.9) \times 10^{25} \mathrm{~s}^{-1}$, yielding an apparent $\mathrm{CO} / \mathrm{H}_{2} \mathrm{O}$ abundance of $0.2 \%-0.5 \%$, the lowest measured in any comet (Weaver et al. 2011). From the prompt CO Cameron band emission a production rate of $Q\left(\mathrm{CO}_{2}\right)>$ $2.0 \times 10^{27} \mathrm{~s}^{-1}$ was inferred from $H S T$.

Measurements on 2010 November 5, of the visible CN (0-0) band obtained with the high-resolution spectrometer UVES of the ESO VLT revealed carbon and nitrogen isotopic abundances. The ${ }^{12} \mathrm{C} /{ }^{13} \mathrm{C}$ ratio was $95 \pm 15$ close to the solar value (89), and the ${ }^{14} \mathrm{~N} /{ }^{15} \mathrm{~N}$ ratio, $155 \pm 25$, was half that of Earth's value (272), similar to the isotopic ratios that are detected in other comets (Jehin et al. 2009, 2010; Manfroid et al. 2009). This data set also yielded ortho-to-para ratios of $\mathrm{NH}_{2}$ and $\mathrm{H}_{2} \mathrm{O}^{+}$ from which spin temperatures of $33 \pm 3 \mathrm{~K}$ and $>25 \mathrm{~K}$ were derived, respectively. Despite the very strong activity shown by the small nucleus with well-defined gaseous emissions from localized jets, the isotopic composition and spin temperatures of two abundant molecules show that comet 103P/Hartley 2 is not different from other comets in the species for which we have measurements (Shinnaka et al. 2011), although the $\mathrm{D} / \mathrm{H}$ observations are still being reduced.

Ground-based high-resolution infrared spectroscopy with NIRSPEC from the W. M. Keck Observatory and the CRIRES instrument at the ESO VLT determined absolute and relative production rates of parent volatiles $\left(\mathrm{H}_{2} \mathrm{O}, \mathrm{CH}_{3} \mathrm{OH}, \mathrm{C}_{2} \mathrm{H}_{6}, \mathrm{NH}_{3}\right.$, $\mathrm{HCN}, \mathrm{H}_{2} \mathrm{CO}$, and $\mathrm{HC}_{3} \mathrm{~N}$ ) and their coma spatial distributions from 2010 July-December $(r=1.62-1.26$ AU; Dello Russo et al. 2010; Mumma et al. 2010). Changes in production rates up to a factor of two were seen in these species on timescales of days, as well as changes up to $20 \%$ on short timescales (hr), which were related to both rotation of the nucleus and changes in the intrinsic outgassing rate (Mumma et al. 2011). On the night of encounter, overall gas and dust production increased by $\sim 60 \%$ between 10:49 and 15:54 UT (Dello Russo et al. 2011). The SPeX instrument on the NASA Infrared Telescope Facility (IRTF) also observed the comet between 2010 September 30 and November 17 to monitor the $\mathrm{H}_{2} \mathrm{O}, \mathrm{CH}_{3} \mathrm{OH}$, and $\mathrm{C}_{2} \mathrm{H}_{6}$ gas production between 2 and $4 \mu \mathrm{m}$.

The ESA Herschel Space Observatory (Pilbratt et al. 2010) used its full complement of instruments to observe the far-IR and submillimeter spectrum and to image the thermal dust radiation (at 70-672 $\mu \mathrm{m}$ ) from 2010 October 24 to November 17 as part of a Herschel Guaranteed Time Key program (Hartogh et al. 2009). Approximately $2.5 \mathrm{hr}$ before the EPOXI encounter, images of the dust coma at 70,100 , and $160 \mu \mathrm{m}$ were acquired with the PACS instrument (Poglitsch et al. 2010). The same instrument was used at encounter to map the brightness distribution of three $\mathrm{H}_{2} \mathrm{O}$ lines (at 89.9, 179.5, $180.5 \mu \mathrm{m}$ ), from which a water production rate of $\sim 1.2 \times 10^{28} \mathrm{~s}^{-1}$ is derived. Water maps show excess emission in the tail direction, which might be related to a production from large icy grains accelerated in the antisolar direction by non-gravitational forces (Figure 2(b)). The asymmetric nature of the water emission from the comet is confirmed by measurements obtained later using the SPIRE and HIFI instruments (Griffin et al. 2010; de Graauw et al. 2010).

The comet's outgassing and molecular composition were monitored in the submillimeter and millimeter range from 2010 October 15 through November 9 with the IRAM, JCMT, ARO, and CSO facilities. The $\mathrm{HCN}, \mathrm{CH}_{3} \mathrm{OH}, \mathrm{H}_{2} \mathrm{CO}, \mathrm{H}_{2} \mathrm{~S}, \mathrm{CH}_{3} \mathrm{CN}$, $\mathrm{HNC}$, CS, and HNCO molecules were detected, and upper limits on the abundance of $\mathrm{SO}_{2}, \mathrm{c}-\mathrm{C}_{3} \mathrm{H}_{2}$ and deuterated isotopologues of $\mathrm{HCN}, \mathrm{H}_{2} \mathrm{CO}$, and $\mathrm{H}_{2} \mathrm{O}$ were obtained. Upper limits on the $\mathrm{D} / \mathrm{H}$ ratio derived from $\mathrm{DCN}$ gave $\mathrm{D} / \mathrm{H}<0.01$ (Milam et al. 2011). This is consistent with other cometary values (e.g., D/ $\mathrm{H}=0.002$ in Hale-Bopp (Meier et al. 1998). The Herschel space observatory has detected HDO in comet 103P/Hartley 2, and the data analysis is in progress. At the time of encounter, the HCN production rate was increasing from 1.3 to $1.8 \times 10^{25} \mathrm{~s}^{-1}$ and the mean gas expansion velocity was $0.7 \mathrm{~km} \mathrm{~s}^{-1}$. 
(a)

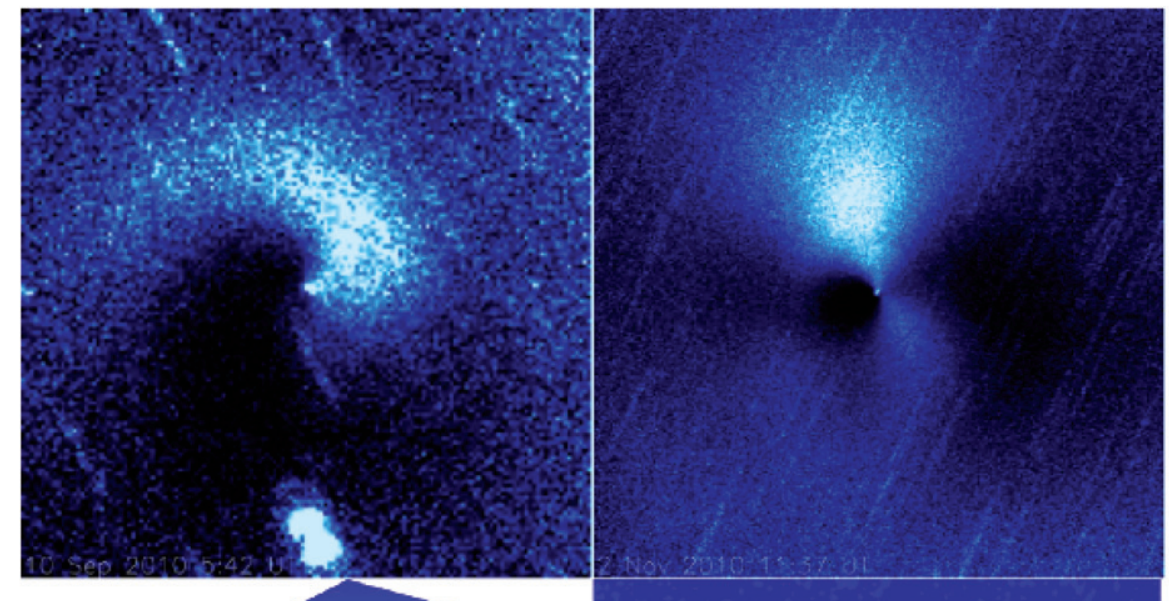

(b)
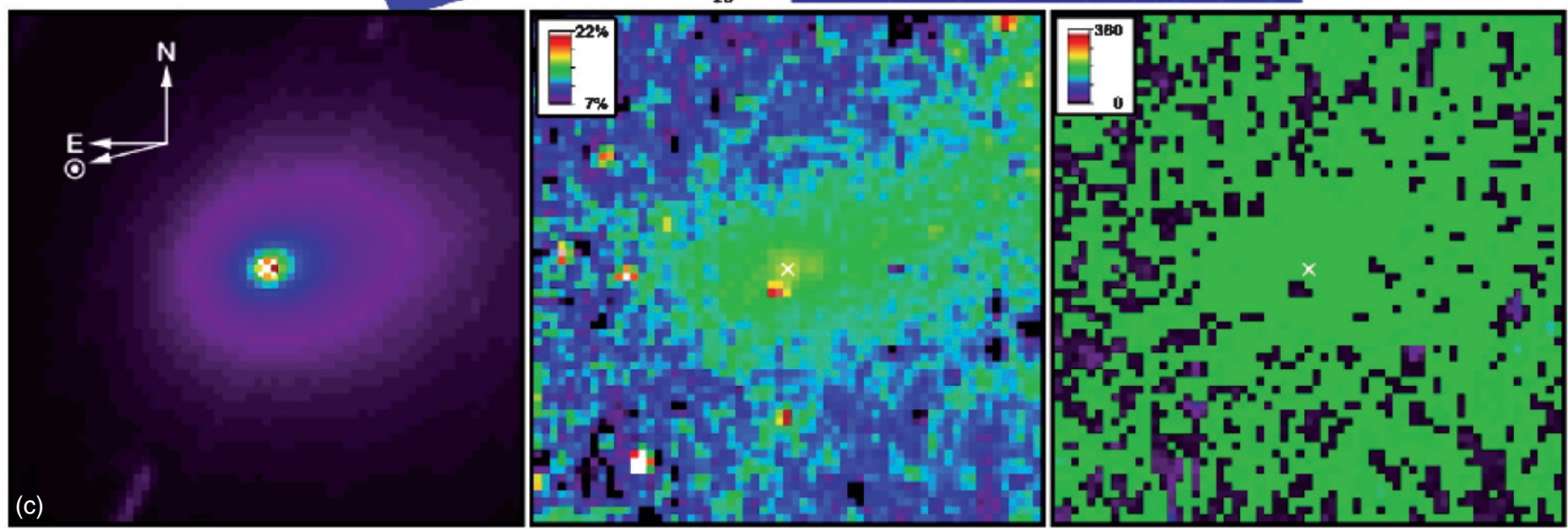

Figure 2. Appearance of the comet at different wavelengths. (a-top) Two enhanced CN images of the comet obtained from the Hall $1.1 \mathrm{~m}$ telescope at Lowell observatory on 2010 September 10 and November 2 showing the jet morphology change. Each image is $\sim 50,000 \mathrm{~km}$ across and oriented so north is up and east is to the left. (b-middle left) Map of the 221-212 water line (1661 GHz) obtained with the PACS on Herschel on November 4.55 UT. The $10^{\prime \prime}$ scale bar corresponds to $1100 \mathrm{~km}$ at the comet's distance. (b-middle right) Image of the dust coma at $70 \mu \mathrm{m}, 100 \times 100$ arcsec in size, obtained with PACS on November 4.47 UT. (c-bottom left) intensity map of the coma in $i$ band on November 4 obtained with the $50 \mathrm{~cm}$ NOAJ telescope polarimetric imager. (c: bottom center) Linear polarization map, (c-bottom right) Position angle of the polarization plane relative to the plane orthogonal to the scattering plane. Scale is $186 \mathrm{~km}_{\text {pixel }}^{-1}$. Arrows show the direction to the Sun.

The Odin submillimeter space observatory (Nordh et al. 2003) monitored the water production rate from 2010 October 29.4-31.7 and November 20.6-21.1. The average production rate inferred from the observation of the $557 \mathrm{GHz}$ water line is $10^{28} \mathrm{~s}^{-1}$ with a $\sim 0.8$ day $25 \%$ amplitude variation. The comet was observed with the Nançay radio telescope from the beginning of 2010 August until the end of 2011 January in order to assess water production by monitoring the $\mathrm{OH} 18 \mathrm{~cm}$ lines. The comet was detected from the end of 2010 September until mid-December, and exhibited day-to-day variability in the water production. Observations of the hydrogen coma were recorded by the SWAN UV camera on the $\mathrm{SOHO}$ spacecraft for three months around the EPOXI flyby from 2010 September 14 through December 12. During early November CN and $\mathrm{OH}$ showed a flux increase with respect to the other species (A'Hearn et al. 2011; Combi et al. 2011). An analysis of these data sets indicates there was significant water production from icy grains. The peak activity in $\mathrm{H}_{2} \mathrm{O}$ measured by $\mathrm{SOHO}$ in 2010 was three times less than that measured with the HST during the 1991 apparition $\left(Q\left(\mathrm{H}_{2} \mathrm{O}\right)\right.$ of $6 \times 10^{28} \mathrm{~s}^{-1}$ in 1991 
Table 2

Mixing Ratios (\%) Relative to Water ${ }^{\mathrm{a}}$

\begin{tabular}{|c|c|c|c|c|c|c|c|c|c|c|}
\hline Tel. & $\lambda$ & $\mathrm{CO}_{2}$ & $\mathrm{CO}$ & $\mathrm{HCN}$ & $\mathrm{C}_{2} \mathrm{H}_{6}$ & $\mathrm{C}_{2} \mathrm{H}_{2}$ & $\mathrm{CH}_{3} \mathrm{OH}$ & $\mathrm{NH}_{3}$ & $\mathrm{H}_{2} \mathrm{CO}$ & Ref \\
\hline EPOXI & IR & 20 & & & & & & & & 1 \\
\hline$H S T$ & UV & $<20$ & $0.15-0.45$ & & & & & & & 2 \\
\hline IRAM JCMT CSO & $\mathrm{mm}$ & & & 0.1 & & & & & & 3 \\
\hline Keck & IR & & & $0.23 \pm 0.01$ & $0.73 \pm 0.02$ & $0.09 \pm 0.01$ & $2.68 \pm 0.17$ & $0.54 \pm 0.07$ & $0.24 \pm 0.04$ & 4 \\
\hline Keck & IR & & & $0.26 \pm 0.02$ & $0.77 \pm 0.04$ & $0.14 \pm 0.01$ & $1.23 \pm 0.06$ & $0.59 \pm 0.06$ & $0.11 \pm 0.01$ & 5 \\
\hline
\end{tabular}

Notes.

a At the time at which activity peaked at $q+10$ days, the production was $\sim 1 \times 10^{28} \mathrm{~s}^{-1}$, but this varied with rotation. There was an outburst on 2010 September 16 during which time the $\mathrm{H}_{2} \mathrm{O}$ production was a little higher.

References. (1) A'Hearn et al. 2011; near maximum; $\mathrm{CO}_{2} / \mathrm{H}_{2} \mathrm{O}$ varies by up to $2.9 x$; (2) Weaver et al. 2011 ; $\mathrm{CO}$ varied by $30 \%$; $\mathrm{CO}$ inferred from $\mathrm{CO}$ Cameron emission. Range represents large uncertainty in mixing ratio. (3) Drahus et al. 2011; HCN varies by $2 x$; (4) Mumma et al. 2011; average from 2010 July to December. Water, ethane, HCN, and methanol vary with rotation, the mixing is independent of rotation; (5) Dello Russo et al. 2011. Average from 2010 November 4 . All but $\mathrm{H}_{2} \mathrm{CO}$ are typical of other comets; $\mathrm{H}_{2} \mathrm{CO}$ is depleted. The difference in methanol production ratio is due to using different $v_{3} Q$-branch $g$-factors.

September; Weaver et al. 1994) and $2 \times 10^{28} \mathrm{~s}^{-1}$ in 1998 January (H. Weaver, private communication), thus there seems to be a clear monotonic downward trend in water production in the past decade. Table 2 presents a summary of the various mixing ratios reported here and in the subsequent papers. It should be noted that for interpretation of the differences in mixing ratios, the source papers need to be consulted. The mixing ratios may have systematic uncertainties introduced by using different excitation models, through the treatment of optical depth effects, coma model uncertainties, and temporal variations.

\section{DUST PROPERTIES}

Observations from a suite of telescopes characterized both the small and large dust grain components in the comet. The WISE (Wright et al. 2010) imaged 103P/Hartley 2 during 2010 May 10-11, when it was at $r=2.3 \mathrm{AU}$, and imaged a dust trail extending more than $20 \operatorname{arcmin}\left(1.8 \times 10^{6} \mathrm{~km}\right)$. Subsequent modeling suggested that the dust grains had radii from 0.5 to $6 \mathrm{~mm}$ with a relatively steep mass size distribution (Bauer et al. 2010).

The dust coma observed on 2010 November 4 with Herschel shows a similar asymmetry as the gas (Figure 2(b)). The dust production rate inferred from the PACS images is preliminary, as it depends on the dust size distribution, which is not yet constrained. Using the dust model described in Bockelee-Morvan et al. (2010), dust production rates in the range $250-750 \mathrm{~kg} \mathrm{~s}^{-1}$ were determined for size indices at the nucleus of 3.5-3.7, and a dust-to-gas production ratio of 1.5-2 was determined. The comet was observed by SPIRE as well in photometric bands at 150,250 , and $500 \mu \mathrm{m}$, and the presence of strong submillimeter emission confirms that large grains were present, in agreement with the in situ measurements (A'Hearn et al. 2011).

Mid-IR narrowband spectrophotometry $(7-13 \mu \mathrm{m})$ of the comet was obtained at 11 epochs spread over 2010 October 31-November 5, at the NASA IRTF with the MIRSI instrument (Deutsch et al. 2003; Kassis et al. 2008). The $11.6 \mu \mathrm{m}$ flux density measured in a $3^{\prime \prime}$ radius varied with time (mean = $\left.(1.54 \pm 0.05) \times 10^{-13} \mathrm{~W} \mathrm{~m}^{-2} \mu \mathrm{m}^{-1}\right)$. The max-to-min ratio on all nights is 1.45 , although it is not clear from the data set that the full range of the mid-IR light curve was measured. These results agree with the mid-IR variability observed about a week earlier with the Berkeley Automated Supernova Search (BASS) instrument (Sitko et al. 2010). The mean strength of the $10 \mu \mathrm{m}$ silicate emission feature as measured by the ratio $\left(F_{9.8}+F_{11.6}\right) /$ $\left(F_{7.7}+F_{12.3}\right)$ did not significantly vary $($ mean $=1.19 \pm 0.03)$.
Mid-IR observations from the Subaru $8 \mathrm{~m}$ telescope (with COMICS) on 2010 October 23 and continuing from the GeminiSouth Telescope from November 5 through December 13, showed an extended coma $10^{\prime \prime}$ in the antisunward direction. A silicate feature was continuously present throughout this time period $\sim 20 \%$ above the continuum (Figure $3(\mathrm{a})$ ). The dust production rate varied as the nucleus rotated but the dust composition did not appear to be significantly varying, suggesting either that the activity was dominated by a single active area, or that all active areas have similar dust properties. Although the thermal emission from the nucleus has yet to be considered, the silicate feature is similar in strength to other Jupiter-family comets and to previous observations of comet 103P/Hartley 2 (Kelley \& Wooden 2009).

Near the time of closest approach to Earth (2010 October 22) data were obtained using several instruments at the NASA IRTF facility (BASS, SPeX, and the guide camera) to obtain the dust spectral energy distribution (SED) from 0.4 to $13 \mu \mathrm{m}$ (Sitko et al. 2011). The SED was modeled with a combination of scattered light and a thermal grain component with amorphous silicates (pyroxene and olivine compositions), amorphous carbon and crystalline olivine (Figure 3(b)). From this the bolometric albedo was found to be 0.056 , which is considerably lower than the bulk of active comets measured so far.

The comet was observed by the SOFIA (Becklin \& Gehrz 2009) on 2010 December 3 and 7, using the FORCAST camera (Adams et al. 2010), at aircraft altitudes of 42,000-43,000 ft, in broad filters centered at $11.1,24.2,31.4$, and $37.1 \mu \mathrm{m}$. The comet was clearly detected at greater than $10 \sigma$ in all filters, with spatial extent up to $30^{\prime \prime}$ in the best $(24.2 \mu \mathrm{m})$ image. The presence of significant long-wavelength emission indicates a substantial population of particles larger than $10 \mu \mathrm{m}$ as shown in Figure 3(b).

Polarization of comet 103P/Hartley 2 dust showed a significant dependence on aperture, increasing as the field of view got smaller. At a phase angle of $\sim 59^{\circ}$ the polarization was $13.2 \%$ ( $~ 9600 \mathrm{~km}$ aperture) in the red continuum $(6840 / 90 A)$ and $10 \%$ in the blue continuum $(4845 / 65 A)$, falling between values typical for dust- and gas-rich comets. However, at smaller apertures ( $\sim 3000 \mathrm{~km}$ diameter) polarization reached $6 \%$ at phase angles of $30^{\circ}$ that is much higher than for dust-rich comets and was typical for the high-polarization comet Hale-Bopp. This would imply a polarization of $\sim 20 \%$ at $59^{\circ}$. The increase of polarization with decreasing nucleocentric distance is also evident from the polarization map in the $I$ filter from the NAOJ on November 4 (Figure 2(c)) which gives an average linear polarization of about 

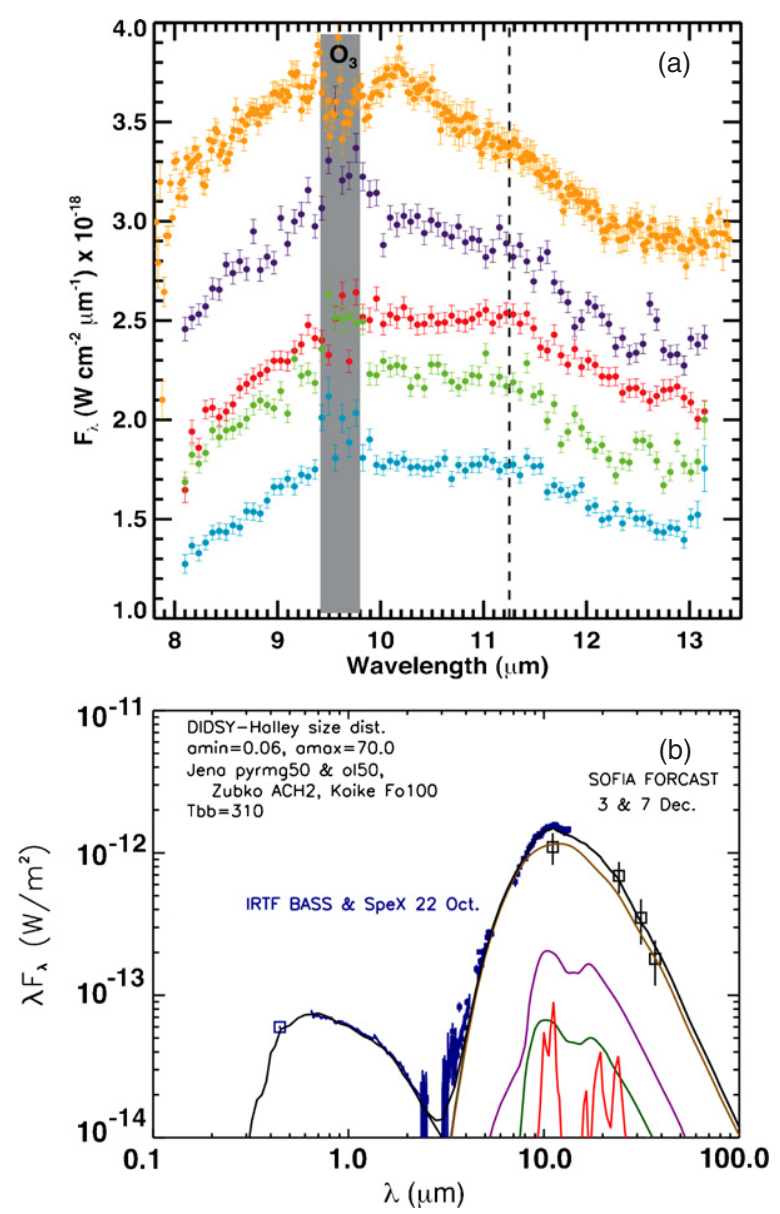

Figure 3. (a) Mid-IR $10 \mu \mathrm{m}$ spectra obtained with COMICS on Subaru during 2010 October and T-ReCS on Gemini-South during November and December showing the evolution of the silicate emission feature of comet 103P/Hartley 2. The spectra were centered on the nucleus region using a 0.33 wide slit (COMICS) and a 0.' 65 wide slit (T-ReCS) and extracted $1^{\prime \prime}$ in the spatial direction for 2010 October 23 (orange), November 5.3 (dark blue), 7.3 (red), 21.3 (green), and December 13.3 (cyan). The October data have been divided by a factor of 2.7 and the December data have been multiplied by a factor of two for the display. The gray area indicates the location and width of the terrestrial ozone absorption bands. The vertical dashed line indicates the wavelength location $(11.2 \mu \mathrm{m})$ of the Mg-rich crystalline olivine feature. (b) The SED of 103P/Hartley 2 obtained with the BASS-SPeX IRTF data and a model used to extract the bolometric albedo. The thermal emission has a component from carbonaceous and silicate grains (colored lines) with $T=295 \mathrm{~K}$ and a warmer blackbody. Longer IR data from SOFIA obtained in early December are also shown.

$15 \%-16 \%$ within $5^{\prime \prime}(\sim 550 \mathrm{~km})$ of the image center. When there is a high gas contamination that increases with increasing distance from the nucleus due to the increase of the amount of gas relative to the amount of dust, this can decrease the values of polarization due to depolarization by gas emissions, especially in the blue filter. Such a behavior is typical for low dust-to-gas ratio comets. Near-IR ( $K^{\prime}$ band) polarimetric measurements were made using the IRTF on 2010 October 17.55 and 2010 November 15.21 UT giving a linear polarization of $14.00 \% \pm 0.86 \%$ and $14.45 \% \pm 1.44 \%$, respectively, while the flux density in a 2 .'0 circular aperture varied by a factor of two between those dates. The average IR polarimetric values, extrapolated to the optical 58.8 phases, yield a neutral polarimetric color, although the polarimetric color is red in the visible. Such behavior is typical for cometary dust and indicates that the dust particles act as porous on the scale of $0.4-0.7 \mu \mathrm{m}$ and compact on the scales of 1-3 $\mu \mathrm{m}$ (Kolokolova \& Kimura 2010).
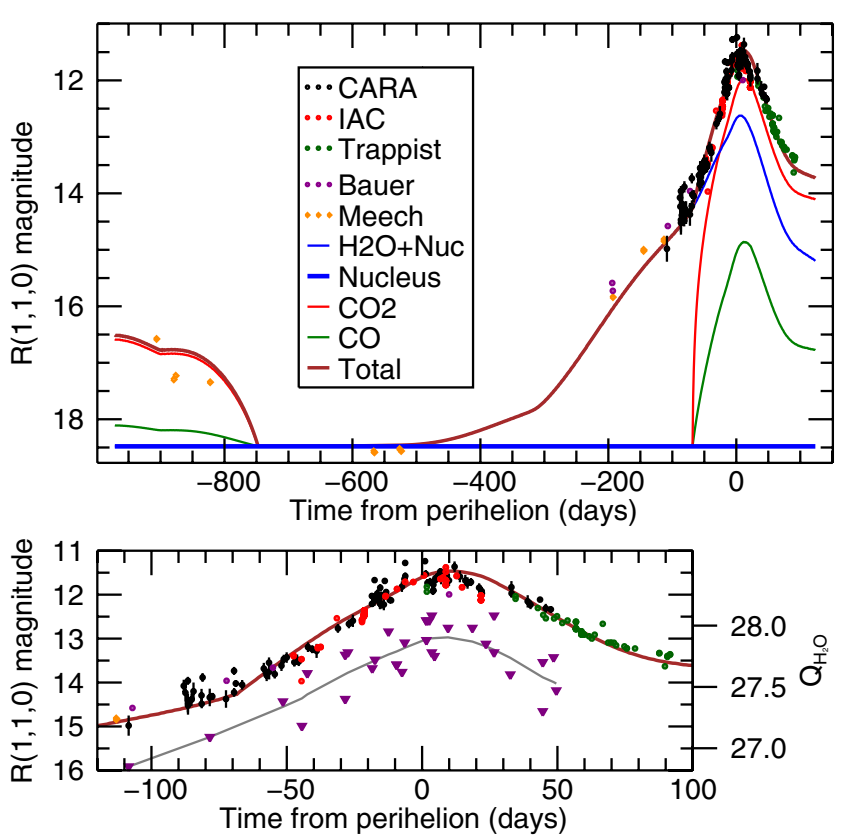

Figure 4. Top: composite photometric light curve of the comet brightness (within $5^{\prime \prime}$ radius aperture) from the TRAPPIST telescope (green), the CARA consortium (black), the IAC-80 telescope (red), and data from Mauna Kea (orange) and Palomar (purple). For comparison, the best-fit $\mathrm{H}_{2} \mathrm{O}$ (blue), $\mathrm{CO}_{2}$ (orange), $\mathrm{CO}$ (green), and total (brown) model light curves are superimposed. The heavy horizontal blue line is the nucleus brightness. This shows water sublimation controlled activity from $r=4.3-1.4 \mathrm{AU}$, at which point $\mathrm{CO}_{2}$ outgassing from jets began to dominate $(\sim q-60$ day) through perihelion, remaining active out beyond the date of aphelion. Bottom: enlargement of the light curve for 250 days around perihelion comparing the optical data and model fit (brown curve) with the water production rates (purple triangles), showing a peak in both dust and water production $\sim 10$ days after perihelion.

\section{HELIOCENTRIC ACTIVITY}

Photometric optical $R$-band data, which measure scattered light from the dust coma, are a sensitive means of monitoring the activity from the comet. In addition to the TRAPPIST robotic telescope data, several groups started to monitor the light curve of the comet for a period of six months, beginning from 2010 July. These included monitoring by a CSIC-IEEC group by using the IAC-80 telescope in the Canary Islands and other medium-sized instruments (Trigo-Rodriguez et al. 2010), the $4 \mathrm{~m}$ SOAR telescope in Chile, the Pan STARRS 1 telescope in Hawai'i, and the CARA project in Italy, a consortium of professional and amateur observers collecting long-term dust production measurements of active comets. The light curve formed from this campaign was combined with the data from 2008 and 2009 and is plotted in Figure 4, which shows both the photometry and the water production rate, both of which peaked $\sim 10$ days post-perihelion. A four-component thermal sublimation model has been generated to estimate the grain flux from the surface driven by a gas flow. The light scattered from the dust is added to that from the nucleus and compared to the data (Meech et al. 1986). The best-fit model has three nucleus volatile components with peak production rates: $\mathrm{H}_{2} \mathrm{O}(2 \%$ fractional surface area active; $\left.3 \times 10^{26} \mathrm{~s}^{-1}\right), \mathrm{CO}_{2}\left(6 \times 10^{26} \mathrm{~s}^{-1}\right)$, and $\mathrm{CO}$ $\left(2 \times 10^{25} \mathrm{~s}^{-1}\right)$. The mixing ratios relative to the water coming from the near surface within a $5^{\prime \prime}$ radius aperture $(\sim 500 \mathrm{~km}$ radius) are $\mathrm{CO}_{2} / \mathrm{H}_{2} \mathrm{O} \sim 100 \%$ and $\mathrm{CO} / \mathrm{H}_{2} \mathrm{O} \sim 4 \%$. However, in order to match the water production reported elsewhere, a component of large icy grains/chunks ejected from the surface must contribute nearly a factor of 20 more sublimating water surface area than the nucleus. The absolute $\mathrm{CO}_{2}$ production 
and inferred presence of large grains is consistent both with the EPOXI spacecraft observations (A'Hearn et al. 2011) and the large grains detected from both Herschel and Arecibo to within the model uncertainties. Adding in the water contribution from sublimating grains, the mixing ratio for $\mathrm{CO}$ becomes $0.2 \%$, in agreement with the HST observations (Weaver et al. 2011) and the value for $\mathrm{CO}_{2}$ is $\sim 7 \%$ in agreement with $H S T$ data from 1991 and 1998 (Weaver et al. 1994; Colangeli et al. 1999). The model also requires that $\mathrm{CO}$ and $\mathrm{CO}_{2}$ activity turns on during 2010 August ( $r \sim 1.4 \mathrm{AU} ; \sim q-75$ days $)$, and off about 200 days post-aphelion $(r \sim 5.6 \mathrm{AU})$. The onset of strong $\mathrm{CO}_{2}$ production is consistent with the time of jet appearance seen in the groundbased imaging data. The ejection of large ice grains has been seen in ice sublimation lab experiments with mixtures of water ice and more volatile materials (Kochan et al. 1998; Bar-Nun \& Laufer 2003). Apart from a coma extension into the dust tail direction, images of the comet showed no strong jet-like features from 2010 March through July, these developed only in 2010 August (Figure 2(a)), coincident with the time that observations started to show a change in the rotation period.

\section{SUMMARY}

1. The nucleus is small, with a low albedo and when inactive the rotation period was observed to be $16.4 \mathrm{hr}$. However, as activity became strong, the rotation rate slowed significantly, possibly driven by either outgassing from the irregular surface or from torques from $\mathrm{CO}_{2}$ jets.

2. The comet had been previously reported as a highly active nucleus with up to $100 \%$ of the surface area active (Lisse et al. 2009; Groussin et al. 2004), but the observing campaign data show that the fractional active nucleus area is normal $(\sim 2 \%)$, but that there is a large halo of icy grains that contribute more $(>90 \%)$ to the total water production rate than does the nucleus (few percentage) at perihelion. Because of the large sublimating ice grain halo, the interpretation of observations as nucleus mixing ratios needs to be approached with caution.

3. A thermal sublimation model shows that the outgassing from the comet began to be detectable at $r \sim 4.4 \mathrm{AU}$, comparable to other comets (Meech et al. 2011) and that just before perihelion $\mathrm{CO}_{2}$ becomes the likely dominant driver of activity. This lasts out to aphelion and the comet remains active after aphelion, on the inbound orbital leg and is likely due to significant contribution of outgassing of $\mathrm{CO}_{2}$ from the interior. This is the first time $\mathrm{CO}_{2}$ has been seen as an important driver for a comet's activity. The outgassing level has been decreasing since the 1991 and 1997 apparitions.

4. The comet appears to be a normal comet in terms of the observed $\mathrm{C}$-chain species, the volatiles that have been detected, the isotopic ratios and the nucleus mixing ratios (Bockelee-Morvan et al. 2004), with the exception of $\mathrm{CO}_{2}$.

5. Water production was correlated with rotation (at near-IR and radio wavelengths). Additionally, there was variability at the $20 \%$ level on short time periods. Neither the groundbased observations nor data from SWAN showed an increase in water production that correlated with the 2010 September CN anomaly seen by the spacecraft.

6. From a thermophysical standpoint, this comet may be a relatively newly introduced object in the inner solar system, given its farther orbit in the past, its physical properties and the abundance and prominence of $\mathrm{CO}_{2}$ ice in contributing to the observed activity. However, more analysis and modeling would be required to assert this inference.
7. Both large and small dust grains are seen, and the grain albedos are low (0.056). From the dust spectroscopy and polarimetry, the physical properties and composition of the comet dust do not appear unusual for a Jupiter-family comet.

Odin is a Swedish-led satellite project funded jointly by the Swedish National Space Board, the Canadian Space Agency, the National Technology Agency of Finland, and the Centre National d'Etudes Spatiales (CNES, France). The Swedish Space Corporation is the prime contractor, also responsible for Odin operations. Herschel is an ESA space observatory with science instruments provided by European-led Principal Investigator consortia and with important participation from NASA. We thank A. Tokunaga, Director of the NASA IRTF, for allocating time for an observing campaign at this telescope. The Faulkes Telescope North contributed to the CARA observing campaign. TRAPPIST is a project driven by the University of Liège, in close collaboration with the Observatory of Geneva, supported by the Belgian Fund for Scientific Research (FNRS) and the Swiss National Science Foundation. The research leading to these results has received funding from the European Community's Seventh Framework Programme (FP7/2007-2013 under grant agreement no. 229517. Faulkes Telescope North contributed to CARA around the flyby period. The CSIC-IEEC and CSIC-IAA teams acknowledge support from grants AYA200801839/ESP and AyA2009-08011 from the Spanish Ministerio de Ciencia e Innovación and PE2007-TIC 02744 from Junta de Andalucía. This work was supported in part by the NASA Planetary Astronomy Program and was performed in part at the Jet Propulsion Laboratory under contract with NASA. This work is also supported in part at The Aerospace Corporation by the Independent Research and Development Program. This work was supported by NASA's Discovery Program through contract NNM07AA99C to the University of Maryland, and in part through the NASA Astrobiology Institute under cooperative agreement no. NNA04CC08A.

\section{REFERENCES}

Adams, J. D., et al. 2010, Proc. SPIE, 7735, 77351

A'Hearn, M. F., Millis, R. L., Schleicher, D. G., Osip, D. J., \& Birch, P. V. 1995, Icarus, 118, 223

A'Hearn, M. F., Schleicher, D. G., Millis, R. L., Feldman, P. D., \& Thompson, D. T. 1984, AJ, 89, 579

A'Hearn, M. F., et al. 2011, Science, in press

Bar-Nun, A., \& Laufer, D. 2003, Icarus, 161, 157

Bauer, J., et al. 2010, IAU Circ., 9179, 1

Becklin, E., \& Gehrz, R. D. 2009, Proc. SPIE, 7453, 745302

Belton, M. J. S., et al. 2011, Icarus, 213, 345

Bockelee-Morvan, D., Crovisier, J., Mumma, M. J., \& Weaver, H. A. 2004, in Comets II, ed. M Festou, H. U. Keller, \& H. A. Weaver (Tucson, AZ: Univ. Arizona Press), 391

Bockelee-Morvan, D., et al. 2010, A\&A, 518, L149

Colangeli, L., et al. 1999, A\&A, 343, L87

Combi, M. R., Bertaux, J.-L., Quémerais, E., Ferron, S., \& Mäkinen, J. T. T. 2011, ApJ, 734, L6

de Graauw, T., et al. 2010, A\&A, 518, L86

Dello Russo, N., Vervack, R. J., Jr., Kawakita, H., \& Kobayashi, H. 2010, IAU Circ., 9171, 1

Dello Russo, N., et al. 2011, ApJ, 734, L8

Deutsch, L. K., Hora, J. L., Adams, J. D., \& Kassis, M. 2003, Proc. SPIE, 4841, 106

Drahus, M., et al. 2011, ApJ, 734, L4

Griffin, M. J., et al. 2010, A\&A, 518, L3

Groussin, O., Lamy, P., Jorda, L., \& Toth, I. 2004, A\&A, 419, 375

Harmon, J. K., Nolan, M. C., Howell, E. S., \& Giorgini, J. D. 2010, CBET, 2515,1 
Harmon, J. K., et al. 2010, IAU Circ., 9179, 1

Hartogh, P., et al. 2009, Planet. Space Sci., 57, 1596

Jehin, E., Manfroid, J., Hutsémekers, D., Arpigny, C., \& Zucconi, J.-M. 2009, Earth Moon Planets, 105, 167

Jehin, E., Manfroid, J., Hutsémekers, D., Guillon, M., \& Magain, P. 2010, CBET, 2589, 1

Kassis, M., Adams, J. D., Hora, J. L., Deutsch, L. K., \& Tollestrup, E. V. 2008, PASP, 120, 1271

Kelley, M. S., \& Wooden, D. H. 2009, Planet. Space Sci., 57, 1133

Knight, M., Schwieterman, E., \& Schleicher, D. 2010, IAU Circ., 9163, 1

Kochan, H. W., Huebner, W. F., \& Sears, D. W. G. 1998, Earth Moon Planets, 80,369

Kolokolova, L., \& Kimura, H. 2010, A\&A, 513, A40

Lisse, C., et al. 2009, PASP, 121, 968

Manfroid, J., et al. 2009, A\&A, 503, 613

Meech, K. J., Jewitt, D., \& Ricker, G. R. 1986, Icarus, 66, 561

Meech, K. J., et al. 2009, AAS DPS, 41, \#20.07

Meech, K. J., et al. 2011, Icarus, 213, 323

Meier, R., et al. 1998, Science, 279, 1707

Milam, S. N., Charnley, S. B., Chuang, Y.-L., Coulson, I. M., \& Remijan, A. R. 2011, 42nd LPI Contrib., 1847
Mumma, M. J., et al. 2010, IAU Circ., 9180, 1

Mumma, M. J., et al. 2011, ApJ, 734, L7

Nordh, H. L., et al. 2003, A\&A, 402, L21

Pilbratt, G. L., et al. 2010, A\&A, 518, L1

Poglitsch, A., et al. 2010, A\&A, 518, L2

Samarasinha, N., Mueller, B. E. A., A'Hearn, M. F., \& Farnham, T. L. 2010, IAU Circ., 9178, 1

Shinnaka, Y., Kawakita, H., Kobayashi, H., Jehin, E., Manfroid, J., Hutsemekers, D., \& Arpigny, C. 2011, ApJ, 279, 81

Sitko, M. L., Russell, R. W., Lisse, C. M., Kelley, M. S., Wolff, M. J., \& Hammel, H. B. 2011, 42nd LPI Contrib. No. 1748

Sitko, M. L., et al. 2010, IAU Circ., 9181, 1

Snodgrass, C., Meech, K., \& Hainaut, O. 2010, A\&A, 516, L9

Trigo-Rodríguez, J. M., García-Hernández, D. A., Sánchez, A., Lacruz, J., Davidsson, B. J. R., Rodríguez, D., Pastor, S., \& de Los Reyes, J. A. 2010, MNRAS, 409, 1682

Weaver, H. A., Feldman, P. D., A'Hearn, M. F., Dello Russo, N., \& Stern, S. A. 2011, ApJ, 734, L5

Weaver, H. A., Feldman, P. D., McPhate, J. B., A'Hearn, M. F., Arpigny, C., \& Smith, T. E. 1994, ApJ, 422, 374

Wright, E. L., et al. 2010, AJ, 140, 1868 